\title{
Performance test of new MPPC for a new neutrino detector WAGASCI
}

\author{
F. Hosomi ${ }^{*}{ }^{1}$ N. Chikuma, ${ }^{1}$ A. Izmaylov, ${ }^{1}$ T. Koga, ${ }^{1}$ M. Yokoyama, ${ }^{1}$ M. Antonova, ${ }^{2}$ \\ M. Khabibullin, ${ }^{2}$ A. Khotjantsev, ${ }^{2}$ Y. Kudenko, ${ }^{2}$ A. Mefodiev, ${ }^{2}$ O. Mineev, ${ }^{2}$ \\ T. Ovsiannikova, ${ }^{2}$ S. Suvorov, ${ }^{2}$ N. Yershov, ${ }^{2}$ A. Bonnemaison, ${ }^{3}$ R. Cornat,${ }^{3}$ \\ O. Drapier, ${ }^{3}$ O. Ferreira, ${ }^{3}$ F. Gastaldi, ${ }^{3}$ M. Gonin, ${ }^{3}$ Th. A. Mueller, ${ }^{3}$ A. Blondel, ${ }^{4}$ \\ F. Cadoux ${ }^{4}$ Y. Favre, ${ }^{4}$ E. Noah, ${ }^{4}$ M. Rayner, ${ }^{4}$ T. Hayashino, ${ }^{5}$ A. K. Ichikawa, ${ }^{5}$
} A. Minamino, ${ }^{5}$ K. Nakamura, ${ }^{5}$ T. Nakaya, ${ }^{5}$ B. Quilain, ${ }^{5}$ J. Harada, ${ }^{6}$ K. Kin, ${ }^{6}$ Y. Seiya, ${ }^{6}$ K. Yamamoto, ${ }^{6}$ Y. Hayato ${ }^{7}$

${ }^{1}$ The University of Tokyo, Department of Physics, Tokyo, Japan

${ }^{2}$ Institute for Nuclear Research of the Russian Academy of Sciences, Moscow, Russia

${ }^{3}$ Ecole Polytechnique, IN2P3-CNRS, Laboratoire Leprince-Ringuet, Palaiseau, France

${ }^{4}$ University of Geneva, Section de Physique, DPNC, Geneva, Switzerland

${ }^{5}$ Kyoto University, Department of Physics, Kyoto, Japan

${ }^{6}$ Osaka City University, Department of Physics, Osaka, Japan

${ }^{7}$ The University of Tokyo, Institute for Cosmic Ray Research, Kamioka Observatory, Kamioka, Japan E-mail: hosomi@hep.phys.s.u-tokyo.ac.jp

\begin{abstract}
We have been developing a new neutrino detector named WAGASCI, to measure the cross section ratio of neutrino interaction with water and plastic targets at the J-PARC neutrino beamline. We use newly developed crosstalk suppression type Multi-Pixel Photon Counters (MPPCs) by Hamamatsu to detect the light emitted from scintillators in the WAGASCI detector. The new MPPC has an order of magnitude lower dark noise rate and crosstalk rate than previous MPPCs, and can be operated with higher over voltage, which results in higher photon detection efficiency (PDE). In order to realize a compact readout with a large number of channels, we developed an array of 32 MPPCs. A measurement system to test a large number of MPPCs is being developed.
\end{abstract}

International Conference on New Photo-detectors, PhotoDet 2015

6-9 July 2015

Moscow, Troitsk, Russia

* Speaker. 


\section{Introduction}

Neutrino oscillations have been intensively studied by many experiments for more than fifteen years. The T2K experiment is one of the long baseline neutrino oscillation experiments and is using the J-PARC neutrino beam in Japan. Its far detector, Super-Kamiokande, uses water as target material, while the near detectors use mainly plastic as a target. The use of different target materials is one of the major systematic uncertainties in the T2K experiment [1]. We have been developing a new neutrino detector named WAGASCI (Water Grid And Scintillator detector) that is placed in the J-PARC neutrino beamline. The goal of this project is to measure the charged current cross section ratio between water $\left(\mathrm{H}_{2} \mathrm{O}\right)$ and hydrocarbon $(\mathrm{CH})$ with $3 \%$ accuracy [2].

The optical crosstalk suppression type Multi-Pixel Photon Counters (MPPCs) by Hamamatsu are newly developed and used in the WAGASCI detector. The dark noise rate, optical crosstalk, gain, and relative photon detection efficiency (PDE) of the new MPPC has been measured. We also developed an array of 32 MPPCs to realize a compact readout. It is important to measure the basic characteristics of MPPCs before installation in the detector. Therefore, a measurement system to test a large number of MPPCs has been developed.

\section{WAGASCI experiment}

\subsection{Experimental configuration}

The WAGASCI detector will be installed in the neutrino monitor hall at J-PARC. The T2K experiment employs the off-axis neutrino beam method, which means that the neutrino energy depends on the angle relative to the beam axis and source. The location of the WAGASCI detector will be at $1.6^{\circ}$ off-axis, where the neutrino energy spectrum is similar to that in $\mathrm{T} 2 \mathrm{~K}$, at $2.5^{\circ}$ offaxis. The neutrino energy spectrum peaks at around $600 \mathrm{MeV}$.

\subsection{Detector design}

The WAGASCI detector consists of two parts, a central detector and surrounding muon range detectors (MRDs) as shown in Fig. 1 The central detector is a neutrino interaction target made of $\mathrm{H}_{2} \mathrm{O}$ and $\mathrm{CH}$. The mass of the $\mathrm{H}_{2} \mathrm{O}$ and $\mathrm{CH}$ targets is 1 ton each, and the outer dimension of the central detector is $1 \times 1 \times 2 \mathrm{~m}^{3}$. The $\mathrm{H}_{2} \mathrm{O}$ and $\mathrm{CH}$ targets are arranged in alternating sequence along the beam axis in order to reduce the difference of the neutrino fluxes and the acceptance of MRDs in each target material, because these difference causes systematic uncertainty in the measurement of cross sections.

The central detector also contains 3-mm thick scintillators assembled into a 3D grid-like structure as shown in Fig. 2. The grid-like structure are composed of several layers and arranged in the order of $x$ layer, grid layer, $y$ layer, and grid layer. This structure enables us to reconstruct the track of charged particles produced by neutrino interaction in the target with a large acceptance. The empty space enclosed by the scintillators is filled with the neutrino target materials, $\mathrm{H}_{2} \mathrm{O}$ or $\mathrm{CH}$. The scintillation light is collected by a wavelength shifting (WLS) fiber that is coupled to a MPPC. If neutrinos interact with scintillators in the water target, it causes background events. That is why the thin scintillators are used in order to maximize the ratio of water mass to total mass in a 


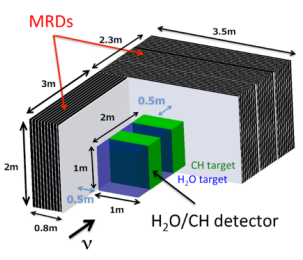

(a) $3 \mathrm{D}$ view

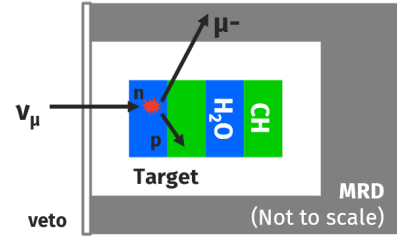

(b) Top view

Figure 1: The configuration of WAGASCI detector.

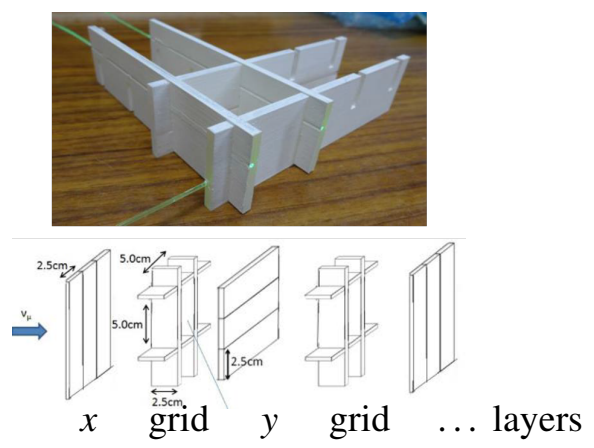

Figure 2: The 3D grid-like structure of the 3-mm thick scintillators.

Table 1: Three types of MPPC measured in this study. The size of pixel is $50 \mu \mathrm{m}$ for all types.

\begin{tabular}{llll}
\hline Type number & Features & Number of pixels & Photosensitive area \\
\hline S10362-11-050C & First generation MPPC & 400 & $1 \times 1 \mathrm{~mm}^{2}$ \\
S12825-050C & Afterpulse suppression & 667 & $1.3 \times 1.3 \mathrm{~mm}^{2}$ \\
S13081-050CS(X1) & Crosstalk suppression & 667 & $1.3 \times 1.3 \mathrm{~mm}^{2}$ \\
\hline
\end{tabular}

fiducial volume up to 79\%. In order to obtain sufficient light yield, newly developed MPPCs with low optical crosstalk and high PDE will be used in the central target.

The MRDs are located on the left side, the right side, and downstream of the central target. They consist of 3 or 6-cm thick iron plates and 7-mm thick scintillators. The MRDs can measure the momentum of muons produced by neutrino interaction up to $1 \mathrm{GeV} / \mathrm{c}$. A magnetic option for the downstream MRD, Baby-MIND, is considered.

\section{Multi-Pixel Photon Counters}

\subsection{Development of MPPC}

The T2K experiment has been using around 56000 MPPCs of the S10362 type by Hamamatsu in their near detectors since 2009. Since then, the performance of MPPC has been significantly improved. The afterpulse and dark noise suppression type (S12825) was released in summer 2013. Then, the optical crosstalk suppression type (S13081) was released in winter 2014. Table 1 summarize some features of these three types of MPPCs, which we also measured in this study. The pixel size of each MPPC is $50 \times 50 \mu \mathrm{m}^{2}$. Figure 3 shows the waveforms of the MPPCs measured with an oscilloscope. Waveforms corresponding to one photon equivalent (1 p.e.) signal, 2 p.e. signal, and so on can be clearly identified. Figure 4 shows micrographs of the MPPCs.

\subsection{Performance test of MPPCs}

The following performance characteristics of MPPCs are measured; dark noise rate, optical crosstalk, gain, and relative PDE. The dark noise rate and optical crosstalk are measured by count-

\footnotetext{
${ }^{1}$ It is same type as used in T2K near detectors (S10362-13-050C) but has different photosensitive area.
} 


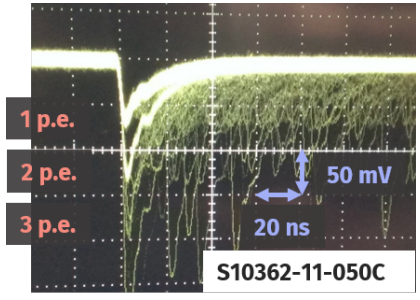

(a) First generation MPPC

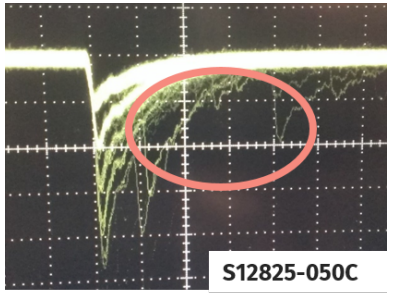

(b) Afterpulse suppression type

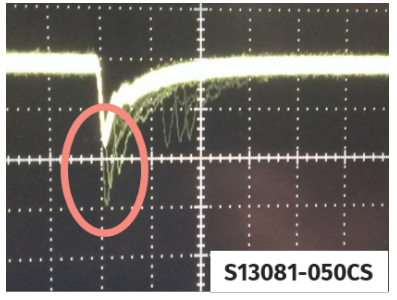

(c) Crosstalk suppression type

Figure 3: The waveforms of the three different MPPCs. Compared to figure (an) afterpulsing is decreased in figure (b), and compared to figure (a) and (b) optical crosstalk is decreased in figure (C).

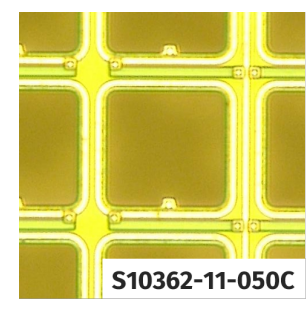

(a) First generation MPPC

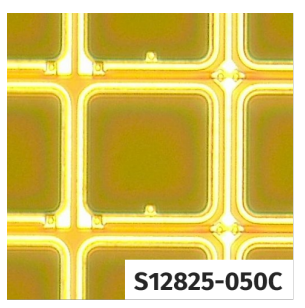

(b) Afterpulse suppression type

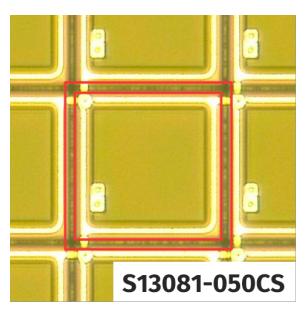

(c) Crosstalk suppression type

Figure 4: The micrographs of MPPCs. There is a trench to prevent crosstalk in the S13081 type MPPC (red box).

ing the rate of discriminated signals with a scaler. The measurement are taken at $20^{\circ} \mathrm{C}$. The dark noise rate is measured by counting the rate of discriminated signals with a 0.5 p.e. threshold level. The dark noise rates normalized to $1 \mathrm{~mm}^{2}$ active area are shown as a function of applied over voltage in Fig. 5a The S12825 type and the S13081 type MPPCs have an order of magnitude lower dark noise rate than the S10362 type MPPC. The significant decrease of the dark noise enables us to operate the new MPPCs with higher over voltage. The optical crosstalk probability is calculated as the ratio of the rate of discriminated signals with 0.5 p.e. and 1.5 p.e. threshold level. Figure 5 b shows the measured optical crosstalk. Compared to the S10362 type MPPC, the S13081 type MPPC has one tenth optical crosstalk, while the S12825 type MPPC has the same optical crosstalk. The substantial suppression of optical crosstalk enables us to lower the signal threshold level in our application.

The gain and relative PDE are measured by injecting light from an LED with a wavelength of $470 \mathrm{~nm}$. The MPPC signals are amplified and recorded with a charge integrating ADC. A $1 \mathrm{kHz}$ clock signal is used to flash the LED and to generate a 120-ns wide ADC gate. The gain calculated from the difference of ADC counts between pedestal events and 1 p.e. events is shown in the Fig.5c Each MPPCs has the same gain for the same over voltage, but the newer MPPCs can be operated over a wider range of operation voltages. With higher over voltage applied, the new MPPC can achieve higher gain and is relatively immune to temperature changes.

When measuring the relative PDE, two MPPCs are measured at the same time in order to reduce the uncertainty of light intensity of LED. The relative PDE is calculated as the ratio of the 


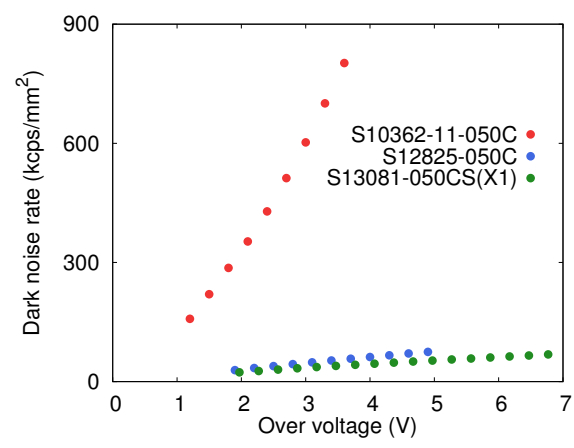

(a) Dark noise rate

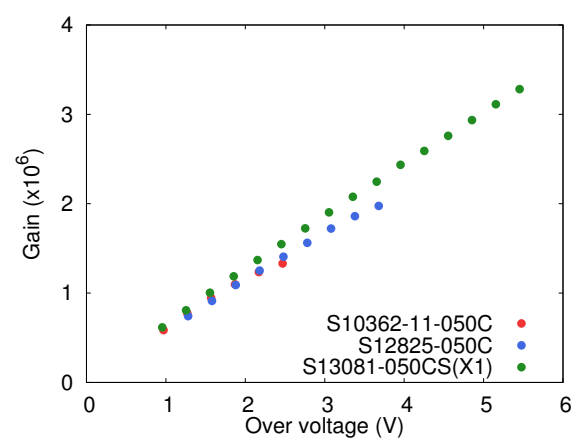

(c) Gain

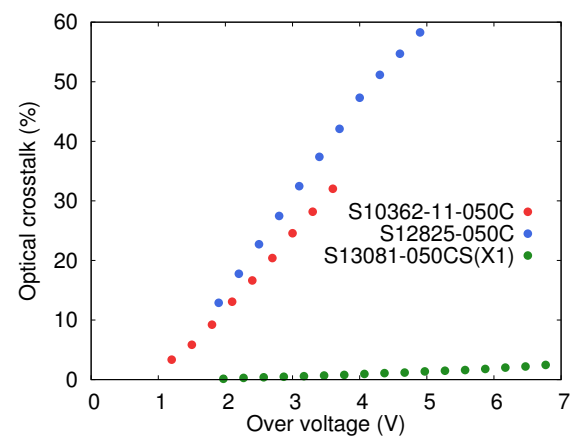

(b) Optical crosstalk

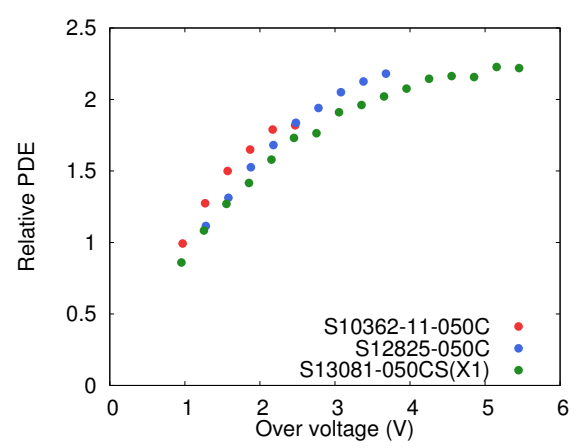

(d) Relative PDE

Figure 5: The results of the performance test for each MPPCs.

Table 2: The comparison of the performance of MPPC. The values are normalized by that of the S10362 type MPPC.

\begin{tabular}{l|r|llll}
\hline Type number & $\begin{array}{l}\text { Applied } \\
\text { over voltage }\end{array}$ & $\begin{array}{l}\text { Dark noise } \\
\text { rate }\end{array}$ & $\begin{array}{l}\text { Optical } \\
\text { crosstalk }\end{array}$ & Gain & $\begin{array}{l}\text { Relative } \\
\text { PDE }\end{array}$ \\
\hline S10362-11-050C & $1.1 \mathrm{~V}^{2}$ & 1 & 1 & 1 & 1 \\
S13081-050CS(X1) & $1.1 \mathrm{~V}$ & 0.077 & N/A & 1.1 & 0.87 \\
S13081-050CS(X1) & $4.0 \mathrm{~V}$ & 0.25 & 0.21 & 3.6 & 1.9 \\
\hline
\end{tabular}

number of photons detected by each MPPCs to the number of photons detected by the S10362 type MPPC with $1.0 \mathrm{~V}$ over voltage. The number of photons detected by the MPPC is calculated as $-\ln \left(P_{0}\right)$, where $P_{0}$ is the fraction of events in which no photon detected. This method is commonly applied when measuring MPPCs and avoids a bias due to afterpulse and optical crosstalk, assuming the true number of detected photons follow the Poisson distribution. Figure 5d shows the measured relative PDE. The newer MPPCs have slightly lower PDE than the S10362 type MPPC for the same over voltage. With higher over voltage applied, however, the newer MPPCs can achieve higher PDE.

The performance of the MPPC is summarized in Table 2, The new MPPC can achieve ten

\footnotetext{
${ }^{2}$ The value of $1.1 \mathrm{~V}$ over voltage is used in the INGRID, one of the T2K near detectors, to operate MPPCs.
} 


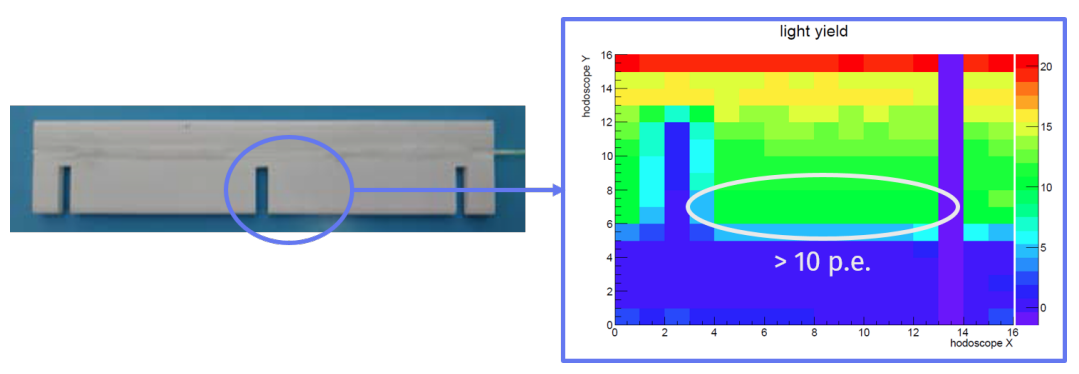

Figure 6: The measured light yield of 3-mm thin scintillator. The positron beam was injected to the circle area in the left figure of scintillator. The light yield of each position is shown right figure.

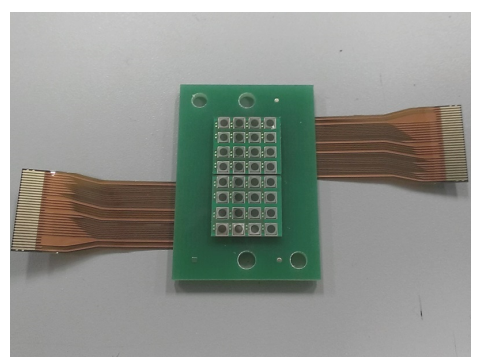

(a) An array of MPPCs

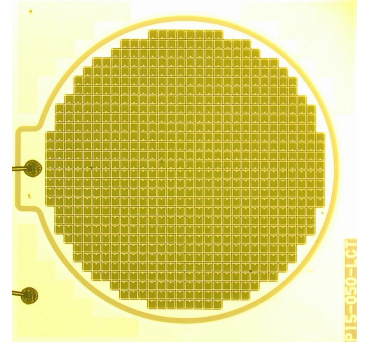

(b) The circular active region of the array of MPPCs

Figure 7: An array of MPPCs.

times lower dark noise rate, more than ten times lower optical crosstalk, higher gain, and higher PDE when higher over voltage is applied. Therefore, the MPPCs used in the WAGASCI detector will be operated with about $4.0 \mathrm{~V}$ over voltage.

The light yield was also checked when combining the new MPPC and 3-mm thin scintillator with slits for a grid-like structure. The scintillation light was collected by a WLS fiber (Y-11) coupled to the S13081 type MPPC operating at $4.0 \mathrm{~V}$ over voltage. Using a $600 \mathrm{MeV}$ positron beam, the position dependency of light yield and the detection efficiency were measured. Figure 6 shows the measured light yield. More than 10 p.e. are collected even from the edge of the scintillator. The detection efficiency was better than $99 \%$ for the whole region of the scintillator with a 1.5 p.e. threshold level.

\subsection{Measurement system development for a large number of MPPCs}

The WAGASCI detector will have a large number of readout channels. In order to realize a compact readout with a large number of channels, we developed an array of MPPCs for the central target of the WAGASCI detector as shown in Fig. 7 The array consists of 32 MPPCs each with $1.5 \mathrm{~mm}$ diameter active region and $50 \times 50 \mu \mathrm{m}^{2}$ pixel size. The array of MPPCs will be used in the central target, while the conventional ceramic packaged MPPCs will be used in the MRDs. The total number of channels used in the WAGASCI detector will be 8126 including 5276 in the arrays of MPPCs and 2750 in the single channel MPPCs. The basic characteristic of the MPPCs will be measured before installation in the detector. A measurement system to test a large number of MPPCs has been developed. 


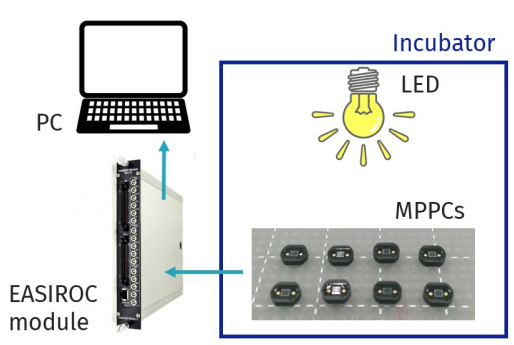

Figure 8: The diagram of measurement setup.

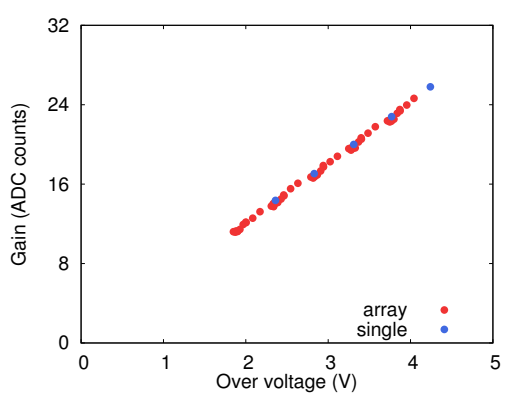

Figure 9: The result of 16 channels measurement.

The dark noise rate, optical crosstalk, gain, and relative PDE will be measured for different temperature and over voltages. The measurement system is designed to test both the arrays and the single channel MPPCs. Figure 8 shows a diagram of the measurement setup. The measured MPPCs and a light source are in a temperature controlled chamber, and the signals from the MPPCs are read out by using EASIROC module, which is a general purpose MPPC readout module controlled via a PC [3]. The measurement system is designed to measure 128 MPPCs simultaneously. Figure 9 shows the preliminary results of measuring an array and a single channel MPPC. The performance of 16 MPPCs could be successfully measured.

\section{Summary}

The WAGASCI experiment aims to measure the charged current cross section ratio between $\mathrm{H}_{2} \mathrm{O}$ and $\mathrm{CH}$ with $3 \%$ accuracy at the J-PARC neutrino beamline. The performance of the newly developed MPPC has been measured. The optical crosstalk suppression type MPPCs used in the WAGASCI detector have an order of magnitude lower dark noise rate and optical crosstalk than earlier devices, and can be operated with higher over voltage, which also results in higher PDE. A sufficient light yield of more than 10 p.e. was achieved when combining 3-mm thin scintillator and the new MPPC. We developed an array of 32 MPPCs for a compact readout with a large number of channels. The total number of channels of the WAGASCI detector is 8126 , and the measurement system to test a large number of MPPCs has been developed.

\section{Acknowledgment}

The work of author (F. Hosomi) has been supported by Advanced Leading Graduate Course for Photon Science (ALPS) at the University of Tokyo.

\section{References}

[1] K. Abe et al. [T2K Collaboration], Phys. Rev. D 91, no. 7, 072010 (2015)

[2] T. Koga et al., JPS Conf. Proc. 8, 023003 (2015)

[3] R. Honda et al., PoS PhotoDet 2012, 031 (2012). 